\title{
INNOVACION DOCENTE MEDIANTE LA GESTION Y PARTICIPACION EN SIMULACROS DE CONGRESOS CIENTIFICOS.
}

\author{
Lucia Aparicio Chofre ${ }^{a}$, Maria Cristina Escamilla ${ }^{b}$ y Carla de Paredes Gallardo ${ }^{c}$ \\ a (Universidad Europea de Valencia y Universidad de Valencia, lucia.aparicio@universidadeuropea.es), b (Universidad Europea \\ de Valencia, mariacristina.escamilla@universidadeuropea.es), c (Universidad Europea de Valencia, \\ carla.deparedes@universidadeuropea.es)
}

\begin{abstract}
The objective of the present work is to show the results obtained in a teaching experience with the students of the 3rd year of the Degree of Criminology and of the Double Degree of Criminology and Psychology of the European University of Valencia, through the performance of a simulation of "congress Scientific "and with the purpose of having contact with the professional reality. The simulacrum was carried out during the 2 nd quarter of the academic year, within the subject of Penitentiary Law and Penology, and consisted in the elaboration of a scientific communication in póster format. This activity was part of the subject, in its practical part, using at first a methodology of training in skills of scientific communication and later of execution of the póster, following established norms with respect to format, structure and extension. The apprentice of the elaboration of this type of scientific communications, allows to carry out works of rigorous form, while pleasant, pleasant and attractive. The result of the work was evaluated by the students as an innovator and motivator in the evaluation surveys. There was a notable improvement in the communicative skills of the participants, which is reflected in the positive evolution of most of them.
\end{abstract}

Keywords: Method, simulacrum, congress, innovation

\section{Resumen}

El objetivo del presente trabajo es mostrar los resultados obtenidos en una experiencia docente con los estudiantes del $3^{\circ}$ curso del Grado de Criminología y del doble Grado de Criminología y Psicología de la Universidad Europea de Valencia, a través de la realización de un simulacro de "congreso científico" y con la finalidad de tener contacto con la realidad profesional. El simulacro se llevó a cabo durante el $2^{\circ}$ trimestre del curso 
académico, dentro de la asignatura de Derecho Penitenciario y Penologia, y consistió en la elaboración de una comunicación científica en formato "póster" y su posterior exposición en salón de actos, diseñado para la celebración de un congreso. Dicha actividad formó parte de la asignatura, en su parte práctica, utilizando en un primer momento una metodología de entrenamiento en habilidades de comunicación científica y pósteriormente de ejecución del póster, siguiendo unas normas establecidas con respecto al formato, estructura y extensión. El aprendizaje de la elaboración de este tipo de comunicaciones científicas, permite realizar trabajos de forma rigurosa, a la vez que amena, agradable y atractiva. El resultado del trabajo fue valorado por el alumnado como innovador y motivador en las encuestas de evaluación. Se produjo una mejora notable en las competencias comunicativas de los participantes, que se refleja en la evolución positiva de la mayoría de ellos.

Palabras claves: competencia, simulacro, comunicación científica, congreso, innovación.

\section{Introducción}

En los últimos tiempos se está produciendo un cambio intenso en la concepción y el objeto de la Educación Superior. La nueva realidad del Espacio Europeo de Educación Superior, propone nuevas metodologías educativas y persigue diferentes objetivos pedagógicos. Esta transformación aboga por un proceso formativo centrado en la adquisición de competencias y en el desarrollo de todo el potencial humano de los estudiantes.

Algunas de las competencias que mayor importancia adquieren son las relacionadas con la esfera comunicativa, especialmente en aquellos estudios o disciplinas en las que la divulgación y la transferencia científica resultan indispensables, siendo este el caso en ciencias sociales. En este sentido, una comunicación científica eficaz requiere el conocimiento y dominio de diversas herramientas de transferencia del conocimiento. Entre ellas, aquellas relacionadas con la exposición en el marco de los congresos científicos, como la comunicación oral (Gonzalez y Barquero, 2012).

Con el cambio metodológico, la Universidad Europea de Valencia pretende reforzar el aprendizaje de los estudiantes de manera flexible y entretenida, fomentando la 
participación del estudiante en el aula, con la finalidad de prepararles para el mundo profesional, por ello considera que el "simulacro de congreso" es una forma de acercarles a la realidad investigadora.

La realización del simulacro de congreso científico es una estrategia didáctica, útil en la adquisición de las competencias y destrezas necesarias para una correcta exposición de unos resultados de una investigación realizada, sabiendo concluir lo importante de dicha temática para finalmente saber plasmarlo en un póster científico, siendo capaces de poder sintetizar todo el estudio realizado así como transmitir, explicar y defender la información del póster. Dicha alternativa de comunicación, ofrece la oportunidad de presentar estudios, proyectos o experiencias, de forma clara y concisa, con la necesaria información y donde el autor puede desarrollar su creatividad y plasmarla de forma permantente (Guardiola, 2010).

En este sentido, la comunicación en formato póster, recoge las aportaciones más interesantes de un congreso. En algunos congresos científicos se han eliminado las clásicas presentaciones en forma de comunicaciones orales, y donde se ha dado especial protagonismo a las contribuciones en formato póster, dejando así las presentaciones orales para conferencias, mesas redondas, seminarios, etc., donde participan los ponentes o autores más reconocidos en cada temática de interés (Guardiola, 2010).

\section{Objetivos}

El objetivo general de este trabajo es desarrollar un recurso formativo e innovador en el proceso de enseñanza universitaria, donde el alumno adquiera las competencias comunicativas del "saber" científico, además del conocimiento de la asignatura concreta donde se trabaja esta actividad. Es imporante tener en cuenta que ahora mas que nunca, nuestra sociedad se articula alrededor de la comunicación, siendo un pilar fundamental de buen hacer en las tareas profesionales o investigadoras siendo cauces eficaces para compartir conocimiento científico.

Los alumnos conseguirán los siguientes objetivos específicos:

- Aprender parte de los contenidos de una asignatura a través de la elaboración de un póster científico.

- Conocer y dominar una herramienta de transferencia del conocimiento.

- Desarrollar la creatividad en relación a unos contenidos, de manera clara, concisa y atractiva. 
- Prácticar habilidades comunicativas en un entorno académico y científico.

- El uso de las tecnologías de la información y comunicación (TICS)

\section{Desarrollo de la innovación}

El presente trabajo se desarrolló con la implementación dentro de una asignatura, de una actividad dirigida a intervenir en un congreso científico simulado. Para conseguir el objetivo de aprendizaje, la actividad se llevó a cabo dentro de la asignatura de Derecho Penitenciario y Penologia del tercer curso del grado de criminología, Derecho y psicología durante el segundo trimestre del curso académico 2015-2016 y 2015-2016 .

El simulacro se realizó con 15 alumnos del $3^{\circ}$ curso del Grado de Criminología y del doble Grado de Criminología/Psicología del curso 2015-2016 y con 11 alumnos del curso 2016-2017. La actividad se incluyó en el segundo trimestre del curso académico 2015-2016 y curso 2016-2017, como ejercicio obligatorio de la asignatura "Penología y Derecho Penitenciario" asignándole un valor del $30 \%$ de la nota final de la asignatura.

El simulacro del Congreso Científico comenzó poniendo en conocimiento por parte del profesor a los alumnos del contenido del proyecto, incluyendo la metodología a usar para el desarrollo del trabajo, que fue la siguiente:

- Fase informativa, donde se establecieron las fechas de entrega de cada fase del póster. Para que dichos plazos fueran recordados por los alumnos y los tuvieran a su disposición en todo momento, se editó un perfil del simulacro en una red social la cual podían consultar los estudiantes en cualquier momento con cualquier dispositivo para poder tener toda la información necesaria del simulacro, véase Figura 1.

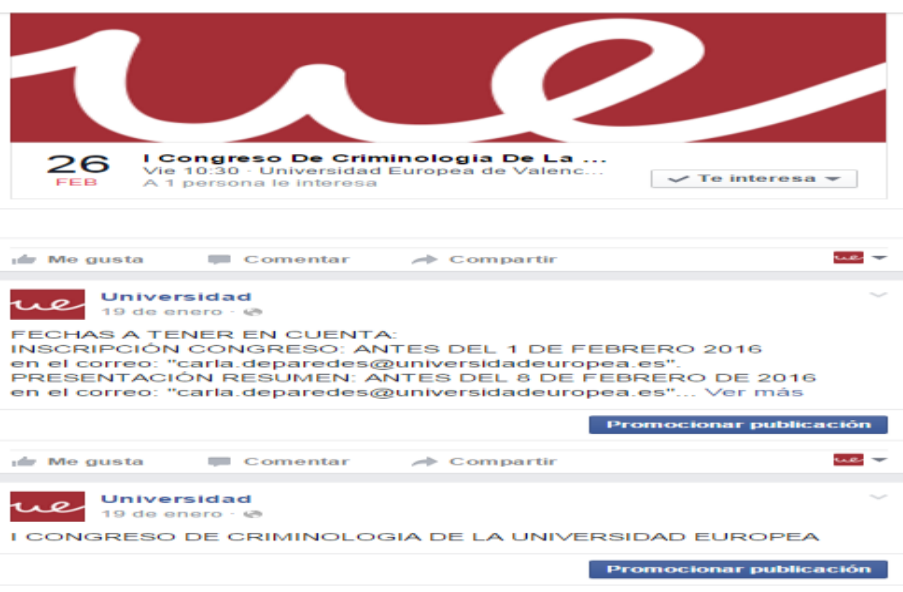

Fig. 1. Información en red social.

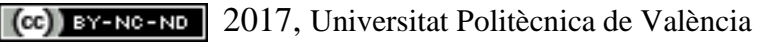


De la misma manera, en esta fase se comunicó a los alumnos los criterios de evaluación de la actividad, siendo un $30 \%$ de la nota final de la asignatura de "Penología y Derecho penitenciario", asignando un 5\% al cumplimiento de los plazos establecidos en cada momento del congreso, por la importancia del cumplimiento de los plazos en Ciencias Sociales, un 5\% a la originalidad del tema elegido así como la investigación realizada sobre el tema electo, otro 5\% a la síntesis realizada en el póster y por último un $15 \%$ a la presentación y defensa del póster ante iguales,

- Fase de elección de la temática: la elección del tema a plasmar en el póster era libre pero siempre dentro del contexto de la asignatura, es decir relacionada con la pena, el derecho Penitenciario, permitiendo la presentación de pósters basados en estudios de datos, de derecho comparado y proyectos de investigación enfocados a la futura realización del Trabajo fin de Grado. Para ello se les recomendó el uso de bibliografía disponible en la biblioteca de la Facultad de Ciencias Sociales, así como de distintas revistas sobre Derecho Penitenciario.

- Fase de inscripción, por parte de los alumnos en el Congreso. Para ello se les facilito un formulario vía virtual, que debían cumplimentar simplemente con sus datos y remitirlo por correo electrónico, dentro del plazo establecido, a una dirección de correo electrónico que se creo exclusivamente para esta actividad.

- Fase de elaboración del póster, en esta fase se ofrecieron pautas a los alumnos para la elaboración del trabajo, referidas tanto a la redacción como a su estructura y creatividad: titulo, autores, centro, introducción, hipótesis y objetivos, metodología (materiales y métodos), resultados, conclusiones, etc. Se ofrecieron explicaciones de las diferentes normas que los congresos solicitan, se vieron ejemplos y plantillas de pósters, además de la revisión de las diferentes características que deben de cumplir a la hora del desarrollo: que sea comprensible, que contenga los puntos esenciales del estudio, proyecto o experiencia, extensión limitada según la organización, claridad, brevedad, lenguaje, tablas, figuras, etc.

- Fase de entrenamiento en habilidades de comunicación científica, en comunicación verbal y no verbal, y sobre el procedimiento a seguir para que la explicación oral sea coherente y justa con un hilo conductor adecuado. 
- Fase presentación en Congreso La fase final del proyecto consistio en el simulacro del congreso científico, los días 26 de Febrero de 2016 y 29 de Marzo de 2017 desde las 10.30 a 12:30 horas respectivamente. Para hacer el simulacro lo mas real posible a la situación real de presentar un póster o comunicación en un congreso, se reservó el salón de actos de la Facultad de Ciencias Sociales y se aconsejó a los estudiantes que acudieran con una vestimenta adecuada para el congreso. Una vez colgado el póster en el sitio asignado a cada autor, se invito a distintos profesores y alumnos para que asistieran como publico, actuando los alumnos como ponentes de las distintas comunciaciones y póster, véase Figura 2.

Dichas comunicaciones se valoraron según la adecuación a la temática de la asignatura, la coherencia en los contenidos, el grado de innovación del trabajo.

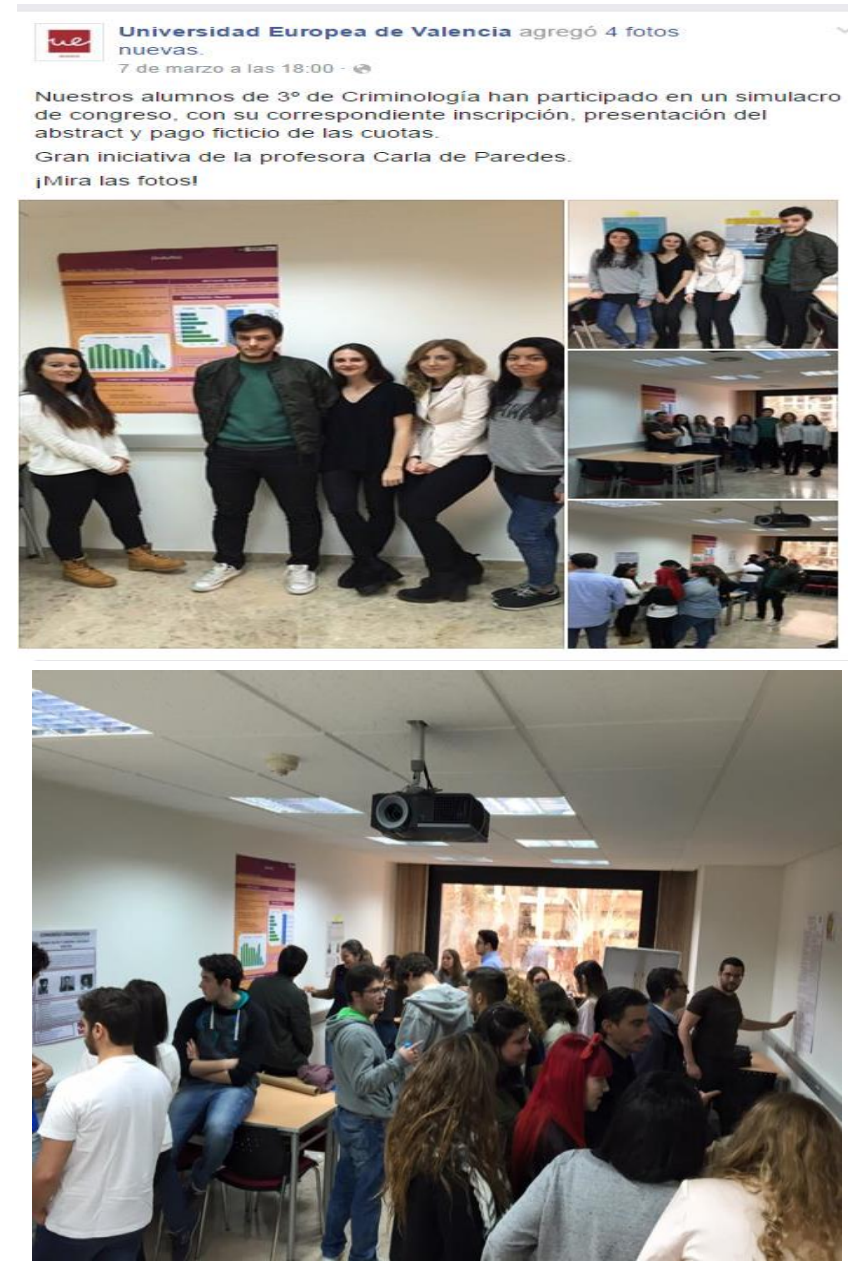

Fig. 2. Imágenes de la experiencia

(cc) EY-NC-ND 2017, Universitat Politècnica de València 


\section{Resultados}

Los resultados obtenidos con esta actividad innovadora fue muy positiva por parte de los alumnos, que manifestaron la iniciativa como novedosa y motivadora al tener un contacto con la realidad investigadora. El proyecto les facilitó conocer los pasos a seguir para contribuir a los congresos científicos profesionales.

Para conocer la opinión de los estudiantes se les paso a todos los participantes una encuesta final de evaluación del simulacro de congreso, el cual consistía en un listado de 15 preguntas cerradas sobre la mejora de las competencias, innovación de la actividad y organización del simulacro, con opciones de respuestas entre A, B, C y D con un valor de total acuerdo, bastante, alguna vez y nada, respectivamente.

El resultado de las encuestas fue mayoritariamente en total acuerdo como actividad innovadora, obteniendo un $80 \%$ de estudiantes con respuestas "A" (sí en total acuerdo) y un $20 \%$ con respuestas "B" (bastante de acuerdo), véase Figura 3.

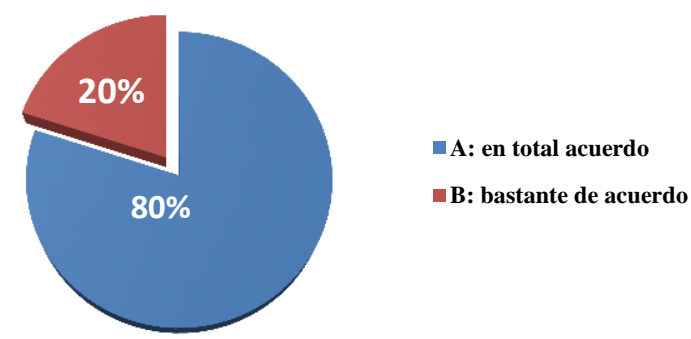

Figura 3. Resultado evaluación de la actividad.

Se observó una mejoraría en la puntuación obtenida por parte de los alumnos en sus competencias comunicativas, entre las dos mediciones que difieren entre sí, 5 semanas, utilizando para ellos los mismos criterios de evaluación.

Los alumnos y los profesores de otras asignaturas que acudieron al simulacro de congreso, manifestaron que les pareció muy enriquecedor el simulacro, no solo por la buena acogida que tuvo entre los alumnos y por los buenos resultados sino también por favorecer una reflexion sobre la innovación docente en general y la comunicación científica en particular, haciendo hincapié del buen trabajo realizado por los estudiantes. 
En el aspecto negativo de las encuestas, señalado por 4 alumnos, es la escasez del tiempo para realizar las tareas encomendadas, siendo bastante alto el nivel de exigencia que require para un alumno enfrentarse a la metodología desconocida, buscar información para realizar la comunicación o póster

\section{Conclusiones:}

La puesta en marcha de esta actividad ofreció diferentes desarrollos de capacidades y aprendizajes al alumno. En primer lugar se vio un gran esfuerzo por sintetizar toda la información recopilada durante el trimestre sobre su tema elegido, eligiendo la información principal que debía figurar en el póster y descartando ideas secundarias. Por lo tanto, los contenidos de la asignatura se interiorizaron desde otra perspectiva y no solo con el estudio. Y en segundo lugar, conocer una herramienta para la comunicación científica, muy eficaz para desarrollar en el mundo academémico y científico.

Por otra parte, se observó una mejoría considerable en las competencias comunicativas, a la hora de explicar entre iguales contenidos criminológicos y jurídicos a otros estudiantes que los desconocen y tener que explicar como concluyen sus ideas. Por todo ello con el entrenamiento en dichas competencias puede afirmarse una gran desarrollo profesional una vez terminados los estudios universitarios.

Por último, un mayor uso de las TICS a la hora de diseñar y elaborar el póster, distinguiendo aquellos alumnos que hacen uso de las TICS de aquellos otros que no las usan y lo hacen a mano.

\section{Referencias}

Galindo, F.; (2009) “El aprendizaje del Derecho y la reforma de Bolonia”. Revista La Ley, Madrid, número 7121. Wolkers Kluwer.

Gonzalez Sanz, J. y Barquero González, A (2012) “ Simulacro de congresos cientifico como entrenamiento en competencias comunicativas". Revista Iberoamericana en Educacion e investigación", Vol. 2, nº 4. pags. 20-28

Guardiola, E. (2010). El póster científico. Serés E, Rosich L, Bosch F, coordinadores. Presentaciones orales en biomedicina. Aspectos a tener en cuenta para mejorar la comunicación. Cuadernos de la Fundación Dr. Antonio Esteve, (20), 85-102.

(c) EY-NC-ND 2017, Universitat Politècnica de València 